\title{
Successful treatment with belimumab of severe systemic lupus erythematosus not responding to standard therapy: a case report
}

\begin{abstract}
Systemic Lupus Erythematosus (SLE) is an autoimmune disease characterized by complex pathophysiology and heterogeneous clinical picture. Belimumab is the first biological therapy licensed for SLE.

Wereport the case of a 24 years old woman affected by a severe form of systemic lupus erythematosus with arthritis, muscle weakness, cervical lymphadenopathy, cutaneous involvement, fever, leukopenia, low complement levels and positivity for anti-dsDNA antibodies. Treatment with high dose steroids, hydroxychloroquine, and mycophenolate mofetil did not induce remission and several disease flares were observed. Therapy with anti-BLyS monoclonal antibody belimumab leads to a fast clinical and laboratory response and to stable remission lasting for 30 months allowing steroid tapering to very low maintenance dose.
\end{abstract}

Keywords: Systemic lupus erythematosus (SLE); Belimumab; Biological therapy

Efficacia di belimumab nel trattamento di un caso di lupus eritematoso sistemico grave non rispondente alla terapia standard

CMI 2016; 10(1): 3-6

http://dx.doi.org/10.7175/cmi.v10i1.1227

\section{INTRODUCTION}

Systemic Lupus Erythematosus (SLE) is a systemic autoimmune disease, whose severity and clinical manifestations are highly variable. SLE pathogenesis is not completely known; however, genetic factors, as well as hormonal and environmental triggers, play a role in the development of the abnormal immune response [1]. SLE diagnosis is based on clinical features and laboratory findings according to current classification criteria $[2,3]$. SLE is a chronic disease and its course is characterized by relapses and remission periods. Therefore, the clinician should set up SLE treatment according to clinical manifestations and severity of flares [4].

Current therapy includes nonsteroidal anti-inflammatory drugs, corticosteroids, anti-malarial agents and immunosuppressive drugs. Belimumab, an anti-B lymphocyte stimulator (BLyS) monoclonal antibody, is the first biologic drug licensed for SLE treatment.

\section{CASE REPORT}

We report the case of a 24 years old woman, who attended our outpatient clinic in February 2012 because of intense joint pain

Why we do describe this case:

- A severe form of SLE with several disease flares

- Non-response to standard therapy

- Fast and long-term remission obtained with anti-BLyS monoclonal antibody belimumab

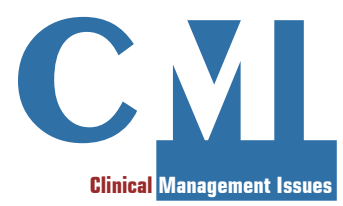

Case report
Department of Internal Medicine, Clinical Immunology Unit, IRCCS-AOU San Martino IST, Genova, Italy
Corresponding author Francesco Puppo Department of Internal Medicine, Clinical Immunology Unit IRCCS-AOU San Martino IST, Genova Viale Benedetto XV, 6 - 16132 Genova, Italy puppof@unige.it

Disclosure

This article has been published with the unconditional support of GlaxoSmithKline S.p.A. 
localized at knees, hands and wrists. She reported a history of asthma and allergic rhinitis (dust mites, dog dander, and olive tree pollen) for which she underwent sublingual immunotherapy in 2010. This therapy was withdrawn because a lack of benefit after two years.

Clinically, she complained of arthritis, muscle weakness, cervical lymphadenopathy and fever associated with leukopenia (white blood cells: $2,640 \times 10 \% / \mu 1)$, low complement levels (C3: $75 \mathrm{mg} / \mathrm{dl}, \mathrm{C} 4: 19 \mathrm{mg} / \mathrm{dl}$ ) and increased erythrocyte sedimentation rate (ESR: $55 \mathrm{~mm} / \mathrm{h}$ ) and C-reactive protein (CRP: $14 \mathrm{mg} / \mathrm{l}$ ) levels. Urinalysis was unremarkable. Immunological investigations showed positivity of antinuclear (ANA, 1:1280 titer with homogenous pattern), antidouble stranded DNA (ds-DNA) and SSARo antibodies; antineutrophil cytoplasm antibodies (ANCA) and antiphospholipid antibodies were negative. Clinical features and laboratory data lead to the diagnosis of systemic lupus erythematosus (SLE) according to current criteria [2,3].

The patient was first treated with prednisone $(1 \mathrm{mg} / \mathrm{kg} /$ day $)$, gradually tapered to $12.5 \mathrm{mg} /$ day, associated with hydroxychloroquine (HCQ) (200 mg twice a day), which did not provide clinical benefit. Then, after a cutaneous flare, in November 2012 HCQ was stopped and mycophenolate mofetil (MMF) (2 g/daily) was started in association with glucocorticoids at variable doses according to clinical needs. Despite these medications, the disease was unsatisfactory controlled.

In May 2013, she presented fever $\left(39^{\circ} \mathrm{C}\right)$, fatigue, intense knees' pain and swelling, and was admitted to our Department of Internal Medicine, Clinical Immunology Unit. Physical examination revealed severe knees' inflammation, tachycardia, and a soft systolic cardiac murmur. Chest X-ray was negative. She also presented cutaneous signs as livedo reticularis and dermographism. Laboratory data showed worsening of leukopenia $\left(1.7 \times 10^{9} / \mu \mathrm{l}\right)$ with severe lymphopenia $(0.3$ $\left.\mathrm{x} 10^{9} / \mu \mathrm{l}\right)$ associated with mild anemia and thrombocytopenia $(62 \times 10 \% / \mu 1)$, abnormal liver function tests (AST $145 \mathrm{U} / 1$; ALT 155 $\mathrm{U} / \mathrm{l}$ ) and increased LDH level (1680 U/1). The severe inflammatory status was confirmed by high CRP $(17.1 \mathrm{mg} / \mathrm{l})$ and ferritin $(29,799 \mu \mathrm{g} / \mathrm{l})$ levels. Blood and urine cultures were negative. A transthoracic Doppler echocardiography showed normal valvular structures without signs of endocarditis as well as normal systolic and diastolic functions. Serology resulted negative for Cytomegalovirus infection but was positive for a recent Epstein-Barr virus infection (positivity of VCA IgM and EBNA 1 IgG antibodies). A computer tomography scan showed liver and spleen enlargement, compatible with the viral infection, and no signs of malignancy. In accordance with these clinical features and laboratory results, we hypothesized an SLE flare triggered by EBV infection. During hospitalization, MMF was
Figure 1. Trend of laboratory parameters before and during belimumab treatment. The vertical line represents the start of belimumab

$\mathrm{ESR}=$ Erythrocyte Sedimentation Rate $\mathrm{CRP}=\mathrm{C}$-reactive protein
Start belimumab

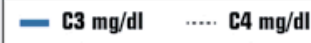

- CRP mg/l - ESR mm/h

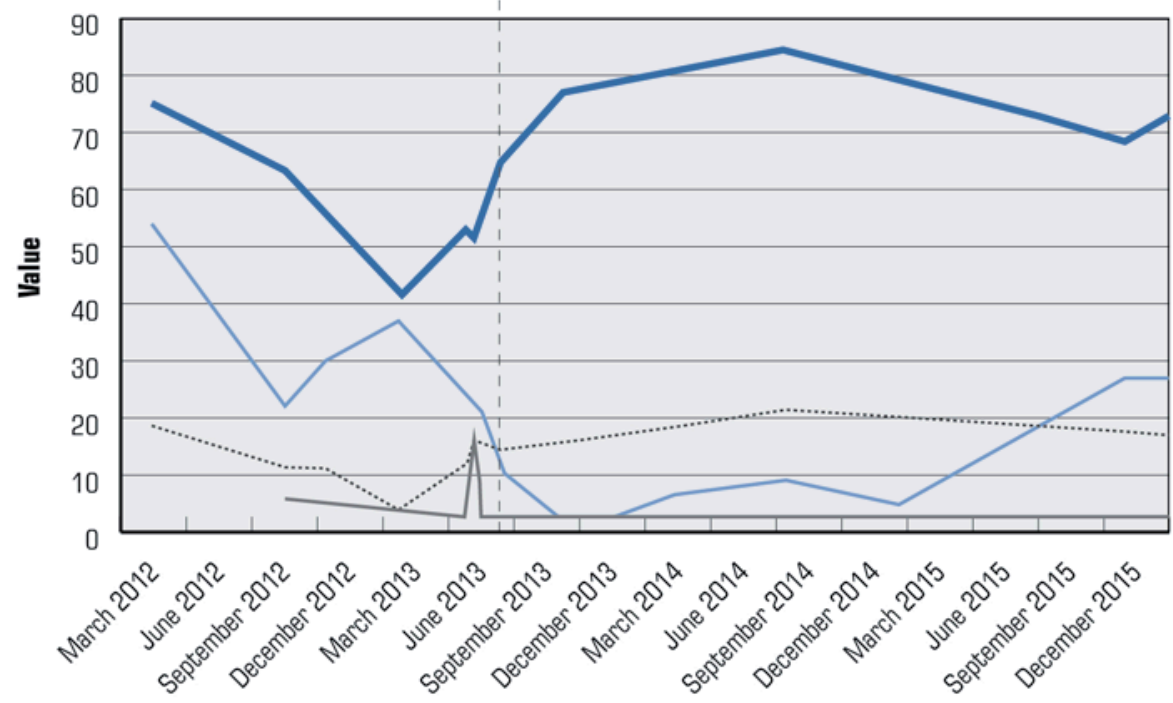




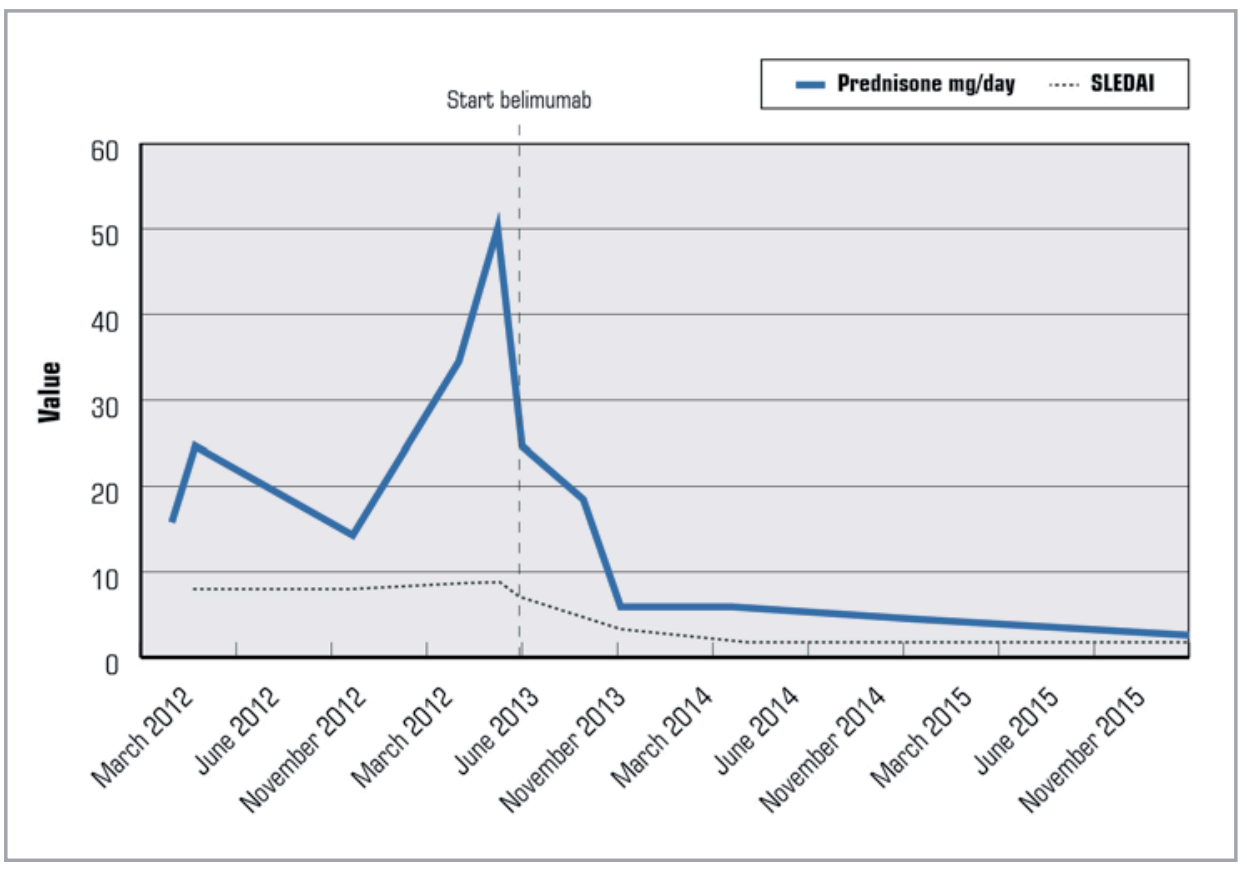

Figure 2. Trend of SLEDAI score (Systemic Lupus Erythematosus Disease Activity Index) and prednisone dose before and during belimumab treatment. The vertical line represents the start of belimumab

stopped, and the patient received methylprednisolone (five $500 \mathrm{mg}$ i.v. infusions) followed by prednisone $1 \mathrm{mg} / \mathrm{kg} /$ day with transient but unsatisfactory clinical improvement (Figure 1).

On the basis of the poor disease control obtained with the previous treatments, we decided to start therapy with the anti-BLyS monoclonal antibody belimumab $(10 \mathrm{mg} /$ $\mathrm{kg}$ ) according to the good results obtained by this biological drug in SLE patients refractory to standard therapy [4-8]. At the time of the first belimumab administration (June 2013) the patient was on treatment only with prednisone $25 \mathrm{mg} /$ daily. No other lupus-specific medications were continued while she was on belimumab. The first three infusions were given 14 days apart and were followed by monthly infusions. We observed a disappearance of arthralgia and constitutional symptoms such as fatigue and fever, 30 days after belimumab was initiated. Hematological parameters and C3 and CRP values reached the normal level after three months of therapy. The amelioration of clinical and laboratory data allowed to decrease the prednisone daily dose from $25 \mathrm{mg}$ to $18.5 \mathrm{mg}$ after 4 months from the beginning of belimumab treatment.

Nowadays, the patient has received 33 belimumab monthly infusions which were well tolerated without any side effect. This therapy leads to a stable remission lasting for 30 months in the absence of disease flares and allowed a further tapering of the prednisone daily dosage to $2 \mathrm{mg}$ (Figure 2).
Main questions the clinician should ask bimself facing such a patient:

- Which are the clinical symptoms and laboratory data suggesting the lack of response to standard therapy?

- How many disease flares should arise to decide to switch from standard therapy to biological drugs?

- Which are the potential side effects of the long-term biological treatment?

\section{DISCUSSION}

Despite the current incomplete understanding of SLE pathogenesis, it is well known that $\mathrm{B}$ cells play a key role in the development of the disease mainly through the production of autoantibodies. Belimumab represents the first biological targeted therapy licensed for the treatment of SLE patients with active disease not responding to conventional therapies [5]. Belimumab is a fully human $\mathrm{IgG} 1 \lambda$ recombinant monoclonal antibody directed against soluble B lymphocyte stimulator (BLyS, also known as BAFF), a member of the tumor necrosis factor (TNF) family. BLyS is involved in lymphocyte survival, activation, and proliferation. In the context of an autoimmune disorder such as SLE, elevated BLyS levels contribute to autoantibodies production and immune-complexes formation leading to inflammatory response and tissue damage. The interaction between belimumab and soluble BLyS prevents the binding of this growth 
factor with its receptors thus decreasing Bcell survival and autoantibodies production.

Since 2013, we have treated with belimumab 10 SLE patients who were refractory to standard therapy. Among them, 7 showed a fast and long-standing response whereas 3 discontinued belimumab for inefficacy. Here we report the successful treatment with belimumab of a young woman with uncontrolled severe active SLE not responding to conventional treatment. Among the wide spectrum of drugs used for SLE therapy, corticosteroids are commonly used. Side effects of long-term corticosteroid intake as hypertension, diabetes, weight gain and osteoporosis are well-known. These side effects could be reduced using a low maintenance daily dose $<7.5 \mathrm{mg}$ [9]. Accordingly, the clinician should consider the best treatment strategy to reduce steroid dose to the lowest possible amount. In our case, belimumab allowed a quick tapering of prednisone without recurrence of the disease. Nevertheless, as belimumab is a relative young drug, more experience is required to ameliorate its management and to know its long-term efficacy and potential side effects.

\section{REFERENCES}

1. Tsokos GC. Systemic lupus erythematosus. N Engl J Med 2011; 365: 2110-21; y of hepatitis B. Semin Liver Dis 2005; 25: 3-8; http://dx.doi.org/10.1056/NEJMra1100359

2. Petri M, Orbai AM, Alarcón GS, et al. Derivation and validation of the Systemic Lupus International Collaborating Clinics classification criteria for systemic lupus erythematosus. Arthritis Rheum 2012; 64: 2677-86; http://dx.doi.org/10.1002/art.34473

3. Kuhn A, Bonsmann G, Anders H, et al. The Diagnosis and Treatment of Systemic Lupus Erythematosus. Dtsch Arztebl Int 2015; 112: 423-32; http://dx.doi.org/10.3238/arztebl.2015.0423

4. Bertsias G, Ioannidis JPA, Boletis J, et al. EULAR recommendations for the management of systemic lupus erythematosus. Report of a Task Force of the EULAR Standing Committee for International Clinical Studies Including Therapeutics. Ann Rheum Dis 2008; 67: 195-205; http://dx.doi.org/10.1136/ard.2007.070367

5. Burness CB, McCormack PL. Belimumab: in systemic lupus erythematosus. Drugs 2011; 71: 2435-44; http://dx.doi.org/doi:10.2165/11208440-000000000-00000

6. Hahn BH. Belimumab for Systemic Lupus Erythematosus. N Engl J Med 2013; 368: 1528-35; http://dx.doi.org/10.1056/NEJMct1207259

7. Wallace DJ, Navarra S, Petri MA, et al. for the BLISS-52 and -76, and LBSL02 Study Groups. Safety profile of belimumab: pooled data from placebo-controlled phase 2 and 3 studies in patients with systemic lupus erythematosus. Lupus 2013; 22 : 144-54; http://dx.doi. org/10.1177/0961203312469259.

8. Ginzler EM, Wallace DJ, Merrill JT, et al. for the LBSL02/99 Study Group. Disease control and safety of belimumab plus standard therapy over 7 years in patients with systemic lupus erythematosus. J Rheumatol 2014; 41 : 300-9; http://dx.doi.org/10.3899/jrheum.121368

9. Ruiz-Arruza I, Ugarte A, Cabezas-Rodriguez I, et al. Glucocorticoids and irreversible damage in patients with systemic lupus erythematosus. Rheumatology (Oxford) 2014; 53:1470-76 http:// dx.doi.org/10.1093/rheumatology/keu148 